\title{
Gravitational Wave Sirens as a Triple Probe of Dark Energy
}

\author{
Eric V. Linder \\ Berkeley Lab \&5 University of California, Berkeley, CA 94720, USA
}

(Dated: October 25, 2018)

\begin{abstract}
Gravitational wave standard sirens have been considered as precision distance indicators to high redshift; however, at high redshift standard sirens or standard candles such as supernovae suffer from lensing noise. We investigate lensing noise as a signal instead and show how measurements of the maximum demagnification (minimum convergence) probe cosmology in a highly complementary manner to the distance itself. Revisiting the original form for minimum convergence we quantify the bias arising from the commonly used approximation. Furthermore, after presenting a new lensing probability function we discuss how the width of the lensed standard siren amplitude distribution also probes growth of structure. Thus standard sirens and candles can serve as triple probes of dark energy, measuring both the cosmic expansion history and growth history.
\end{abstract}

\section{INTRODUCTION}

Observations of distant standardized sources carry information not only on the global cosmology but the signal propagation through the intervening universe. In particular, gravity affects the propagation on all scales, from the global curvature of spacetime to the local inhomogeneities of the mass distribution. In this sense, detecting the acceleration of the cosmic expansion through measuring distances from the luminosity, size, or amplitude of a source is as much gravitational lensing as is the image distortion pattern around massive structures.

While the global spacetime properties determine the distances in a manner fixed by the cosmology, inhomogeneities spread the derived distances about the "truth" or smooth universe value according to a lensing probability distribution function depending on the energymomentum distribution and the convergence and shear it induces in the image. An interesting feature of this probability distribution is the presence of a minimum convergence or maximum demagnification corresponding to an "empty beam" [1]. This cutoff carries cosmological information.

Thus the mean distance measured to standardized sources such as Type Ia supernovae in luminosity or inspiraling supermassive black hole binaries in gravitational wave amplitude (standard sirens [2, 3] ) can probe cosmology, but so can the lensed amplitude distribution through the minimum convergence and the width of the distribution. Gravitational wave sources in particular may be measured to high redshift $z \gg 1$ where lensing effects are quite evident. Standard sirens and candles thus have the potential to be triple probes of cosmology.

In $₫$ II we derive a general expression for the minimum convergence and maximum demagnification, correcting an often used expression in the literature. Implications for gravitational wave standard sirens and supernova standard candles are examined in $\amalg$ III. The use of the minimum convergence as a cosmological probe in its own right is considered in $₫ \mathrm{IV}$, along with its complementarity with distance measurements, and the potential for the lensed distribution width to serve as a growth probe. In the Appendix we briefly discuss effective aver- aging procedures in an inhomogeneous universe and why the standard weak gravitational lensing formula remains valid.

\section{MAXIMUM DEMAGNIFICATION}

For signals on null geodesics such as light rays from supernovae or gravitational waves from inspiraling supermassive black hole binaries, the propagation through the universe can be described by the geometric optics approximation when the wavelength is much smaller than the scales of inhomogeneity. This then leads to the optical scalar equations for a ray bundle [4], whose area defines a luminosity or amplitude distance.

In a globally Friedmann universe, taking Ricci focusing, or convergence, to dominate over rotation and shear leads to the beam equation [5, 6]

$$
\begin{aligned}
\ddot{r} & +[3+q(z)](1+z)^{-1} \dot{r} \\
& +(3 / 2)(1+z)^{-2} r \sum_{w}(1+w) \alpha_{w}(z) \Omega_{w}(z)=0 .
\end{aligned}
$$

Here $r$ is the angular diameter distance (related to luminosity or amplitude distances through redshift factors $1+z$ in a Liouville or phase space density conserving system), an overdot denotes differentiation with respect to $z$, and $q(z)$ is the deceleration parameter. Each energy-momentum component has a dimensionless energy density $\Omega_{w}(z)=8 \pi \rho_{w}(a) /\left[3 H^{2}(z)\right]$, where $H(z)$ is the Hubble parameter, a smoothness parameter $\alpha_{w}=\left(\rho_{w}\right)_{\mathrm{smooth}} / \rho_{w}$, and an equation of state, or pressure to energy density, parameter $w=p_{w} / \rho_{w}$.

Note that only the global properties of the universe enter in the first two terms, while the local, inhomogeneous properties treated by the smoothness parameter $\alpha$ appear in the third term. We can compare two universes with the same global dynamics but different local properties, or two signal paths within the same universe, to find [6]

$$
\begin{array}{r}
r\left(z_{0}, z\right)=\bar{r}\left(z_{0}, z\right)+\int_{1+z_{0}}^{1+z} d u\left[u H(u) / H_{0}\right][\bar{Q}(u)-Q(u)] \\
\times r\left(1+z_{0}, u\right) \bar{r}(u, 1+z),
\end{array}
$$


where $Q(u)=(3 / 2) u^{-2} \sum_{w}(1+w) \alpha_{w}(u) \Omega_{w}(u)$. Note that this is valid for the distance of some source at redshift $z$ from an object (either lens or observer) at redshift $z_{0}$. We will be particularly interested in comparing two signal paths in the same universe so the only difference between the barred distance and the unbarred one comes from different $\alpha$ along the lines of sight.

If we now specialize to the case where there is no contribution of some component $x$ along the entire line of sight, i.e. $\alpha_{x}=0$, this represents the least gravitational focusing, or minimum convergence, possible with respect to the component $x$. That is, one cannot take away more than there is ${ }^{1}$. As shown in [6] (also cf. 7] for the matter only case), the distance $r$ is a monotonic function of $Q$. Therefore the minimum $\alpha$ gives the greatest distance and faintest source, i.e. greatest deamplification relative to a smooth, Friedmann universe.

The convergence is directly related to this distance ratio, since the distance came from considering the beam area. Comparing a given line of sight to the dynamically equivalent smooth universe case (see the Appendix for further discussion), using Eq. (2) we have

$$
\begin{aligned}
& \kappa \equiv 1-\frac{r_{\alpha}}{r_{\mathrm{FRW}}} \\
&=-\frac{3}{2} \int_{1}^{1+z} \frac{d y}{y} \Omega_{m}(y) \frac{H(y)}{H_{0}}(1-\alpha) \\
& \quad \times r_{\alpha}(y) \frac{r_{\mathrm{FRW}}(y, 1+z)}{r_{\mathrm{FRW}}(1+z)} \\
&=-\frac{3}{2} \Omega_{m}(1-\alpha) \int_{1}^{1+z} d y \frac{y^{2}}{H(y) / H_{0}} \\
& \times r_{\alpha}(y) \frac{r_{\mathrm{FRW}}(y, 1+z)}{r_{\mathrm{FRW}}(1+z)},
\end{aligned}
$$

where in the second line we assume for simplicity that only one component is not smooth (e.g. dark energy is smooth but matter can clump) and in the last line we assume $\alpha$ is constant with redshift. These simplifications are not necessary, but for the minimum convergence $\alpha=$ 0 , which is indeed constant (see the Appendix for the general case).

The triplet of distances in Eqs. (4), (5) is often written as $r_{l} r_{l s} / r_{s}$, representing the lens distance, lens-source distance, and source distance. Note that a common practice appears to be to use FRW distances for all three quantities, even when calculating the minimum convergence $\kappa_{\min }$. As we see following [ [6] , the proper expression derived from the optical scalar equations actually distinguishes between the types of distances.

We now give another brief, nonrigorous motivation from the ray deflection equation. (See [8] for more details.) The position of an image that is gravitationally

\footnotetext{
${ }^{1}$ We do not consider $w<-1$, where Ricci focusing can actually go negative. Also, shear acts to increase the Jacobian, i.e. amplification, so the convergence here is indeed the minimum.
}

deflected by an angle $\beta$ is

$$
\vec{\theta} \rightarrow \vec{\theta}-\frac{r_{l s}}{r_{s}} \vec{\beta} .
$$

Here the distances are purely geometric and the ray position is integrated along the unperturbed path, so these distances should correspond to the global spacetime, i.e. be FRW distances. The Jacobian of the transformation (6) gives the convergence and involves

$$
\frac{\partial \beta_{i}}{\partial \theta_{j}}=\frac{\partial \beta_{i}}{\partial x_{j}} r_{l} \rightarrow r_{l} \frac{\partial^{2} \phi}{\partial x_{i} \partial x_{j}}
$$

where $\phi$ is the gravitational potential of the mass perturbation. Because these quantities specifically have to do with the local, inhomogeneous potential, the distance here is not the FRW distance but $r_{\alpha}$. While this may possibly be more an intuitive rationale, we see that both approaches give the same result.

Although using all FRW distances is formally incorrect, under most circumstances the common expression

$$
\kappa_{\text {approx }}=-\frac{3}{2} \Omega_{m} \int_{1}^{1+z} d y \frac{y^{2}}{H(y) / H_{0}} \frac{r_{l}^{\mathrm{FRW}} r_{l s}^{\mathrm{FRW}}}{r_{s}^{\mathrm{FRW}}}
$$

is an excellent approximation, as discussed in the Appendix. The notable exception is when calculating $\kappa_{\min }$, that is Eq. (3), (44), or (5) with $\alpha=0$. Here one explicitly deviates from FRW by requiring that the ray bundle passes through emptied space. Of course this relies on the validity of the Dyer-Roeder approach to light propagation, where the global dynamics is FRW and only Ricci focusing is important. We will be most interested here in the minimum convergence, and as stated, by neglecting shear we obtain a true lower limit. Equation (3), which follows from the optical beam equation and flux conservation in this case [9], then directly leads to Eq. (5). The next section examines the implications of the breakdown of the common formula.

\section{LENSING OF STANDARD CANDLES AND SIRENS}

Convergence can also be expressed in terms of the amplification $\mu$, where the standard amplitude of the candle or siren is taken to be unity, or the demagnification $m$ by

$$
\begin{aligned}
\mu & =(1-\kappa)^{-2} \\
m & =5 \log (1-\kappa) .
\end{aligned}
$$

These expressions do not assume weak lensing but do neglect Weyl shear. The minimum convergence $\kappa_{\min }$ occurs when all the energy density that can be emptied from the beam, i.e. can clump elsewhere, has been so $\alpha=0$ in Eq. (3). Figure 1 shows the values of $\kappa_{\min }$, and the associated $\mu_{\min }$ and $m_{\max }$, as a function of redshift, within a flat LCDM cosmology with $\Omega_{m}=0.3$. 
By $z=3$ one has $\kappa_{\min }=-0.37$ (by definition $\kappa_{\min }$ is negative) and a minimum amplification of $53 \%$ (the standard candle/siren can appear $53 \%$ of its true amplitude) or maximum demagnification of 0.69 mag. For a standardized candle with, say, 0.15 mag dispersion, this is a significant alteration so lensing can have a substantial cosmological impact. A fitting form for $\kappa_{\min }$, accurate to better than 0.01 mag out to $z=10$, is

$$
\kappa_{\min } \approx \begin{cases}-0.067 z^{1.58} & z<3, \\ -0.374-0.182(z-3) & z \geq 3 .\end{cases}
$$

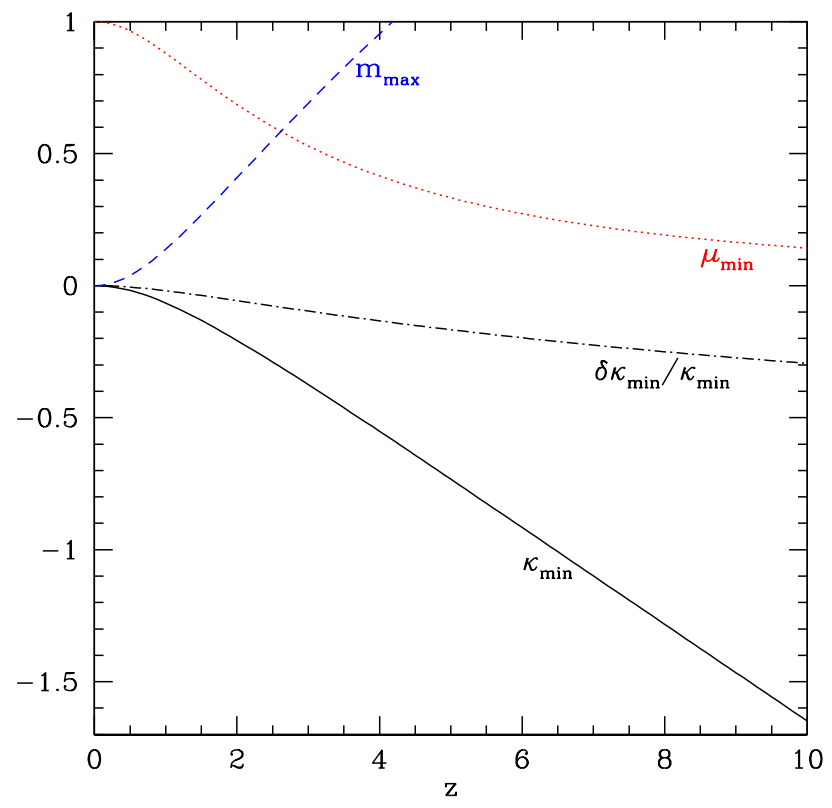

FIG. 1: Lensing causes appreciable effects in standard candle/siren amplitudes, especially at high redshifts. Here the true minimum convergence $\kappa_{\text {min }}$, minimum amplitude $\mu_{\text {min }}$ (as a fraction of the true, standard amplitude), and maximum demagnification $m_{\max }$ are plotted vs. redshift, along with the fractional error $\delta \kappa_{\min } / \kappa_{\min }$ from using the approximate rather than true expression.

Also of importance is the error caused by using the common approximation of Eq. (8). The difference between the correct and approximate expressions for the convergence grows with redshift as shown by the dotdashed line in Fig. 1] This reaches $10 \%$ misestimation of $\kappa_{\min }$ at $z=3$, which is equivalent to a shift in distance scale from misestimating the dark energy equation of state by $\Delta w=0.22$. That constitutes a substantial bias for precision probes of cosmology so the exact expression for convergence, Eq. (3), must be used for accurate cosmology utilizing the minimum convergence. Figure 2 plots the error in maximum demagnification arising from using the approximate expression vs. redshift and shows the equivalent bias that would be induced in $w$. One might also worry that since $\kappa_{\min }$ enters into the universality scaling of the lensing probability distribution function, an error in $\kappa_{\text {min }}$ propagates into all lensing distribution quantities that are derived from the universal distribution via that scaling. However a consistent calculation of lensing distribution functions by, e.g., ray tracing simulations will avoid this.

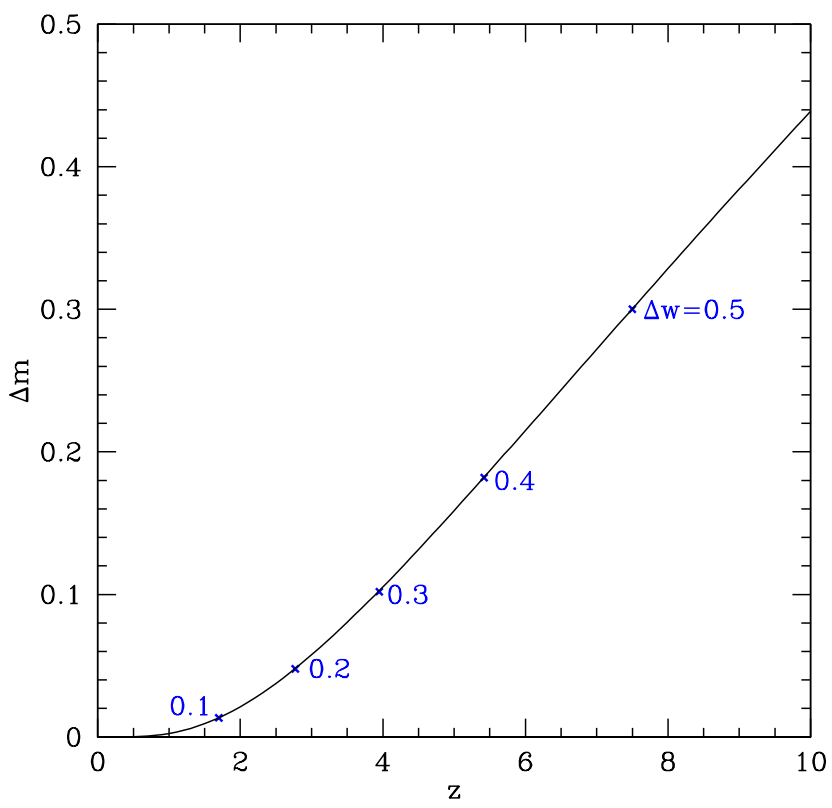

FIG. 2: Magnitude error from using the approximate rather than the true minimum convergence. Such an error leads to a bias in the derived cosmology; labels along the curve show the equivalent offset $\Delta w$ in a constant equation of state, relative to the true value of $w=-1$, caused by using the approximate expression at redshift $z$.

In the next section we investigate the use of the minimum convergence as a source of cosmological information, but first we emphasize that statistically lensing does not affect the mean amplitude measured for the standard candles or sirens. Thus, given sufficient numbers of measurements their role as distance indicators remains intact (see [10] for detailed discussion). As we now see, however, substantial information also exists in the distribution of lensed amplitudes, if well sampled.

\section{COSMOLOGICAL LEVERAGE}

\section{A. Observational Issues}

Measurement of the minimum convergence requires sufficient sources to map out the low amplification end of the lensing probability function. Furthermore, what is actually observed is the convolution of the imperfectly standardized luminosity or strain amplitude function of 
the sources with the lensing amplification. In addition, astrophysical effects such as dust extinction can also dim the luminosity, making it difficult to measure precisely the minimum convergence from the lower limit of the lensing amplification. We briefly discuss these issues (also see [11]) but a detailed treatment awaits future work.

To measure accurately the cutoff $\kappa_{\text {min }}$ one must observe many standard sources to find the lower limit to their amplitudes, $\mu_{\text {min }}$. At high redshift the number of measured sources available will involve the source volume density and rate and the detection threshold for increasingly distant objects. Since gravitational wave amplitudes diminish linearly with distance, rather than going as the inverse square like supernova luminosities, one might expect to detect standard sirens to greater distances. However, observations of their electromagnetic counterparts necessary to measure redshifts might become more difficult. Of course in seeking to measure the most deamplified sources at a given redshift we make the detection more challenging and must ensure Malmquist bias does not arise. A well designed survey can ameliorate these concerns.

If the sources have tightly standardized amplitudes, either luminosity in the case of a standardized candle or metric strain in the case of a standardized siren, then for $1-\mu_{\min }$ much larger than the residual intrinsic dispersion, the value of $\kappa_{\text {min }}$ should be measurable. We see from Fig. 1 that by $z=2$ the minimum convergence corresponds to a demagnification of $0.41 \mathrm{mag}$, much larger than well standardized source dispersions. Thus, $\kappa_{\min }$ for higher redshifts should lie several sigma out on the intrinsic source amplitude probability tail, reducing confusion of source properties with lensing effects. (However, we may not know the source amplitude function well so far from its mean so it is possible that using Gaussian statistics for it will give an overoptimistic assessment.) Since the best gravitational wave sirens can be standardized on an individual basis more precisely than individual supernovae, this should be less of a problem for sirens, though their statistics for mapping the lensing distribution may be poorer. They are also unaffected by residuals to dust extinction corrections that could confuse the interpretation of lensing deamplification.

An important point concerns whether truly empty beams exist in a practical sense. Investigation requires ray tracing through large $\mathrm{N}$-body simulations, convolved with details of survey strategy, and is beyond the scope of this article. However, to develop the idealized promise outlined by the following results, this will need to be done. Two aspects seem hopeful: One is that the sharp cutoff of the magnification probability on the low end, discussed in $₫ \mathrm{IVC}$ means that there may be relatively little error in characterizing $\kappa_{\min }$ even if one measures where the distribution goes to, say, $10 \%$ of maximum amplitude rather than $0 \%$. The other is the possibility that while truly empty beams are rare, there may be a relation between the effective observed $\kappa_{\text {min }}^{\text {obs }}$ and the true $\kappa_{\text {min }}$; if this can be calibrated through N-body sim- ulations then one can still use the technique, albeit at reduced accuracy ${ }^{2}$.

\section{B. Minimum Amplification as Expansion Probe}

Having noted these issues, we approximate them with a highly simplified error model and examine the cosmological information in the minimum convergence, leaving detailed experiment design to the future. Employing the Fisher matrix formalism we assess how measurements of $\kappa_{\min }$ at various redshifts, through either standard sirens or candles, propagate into cosmological parameter constraints. Figure 3 illustrates the sensitivity to the matter density $\Omega_{m}$ and the dark energy parameters $w_{0}$ and $w_{a}$, with the standard time dependent equation of state $w(a)=w_{0}+w_{a}(1-a)$, for a spatially flat, $\Omega_{m}=0.28$ LCDM universe.

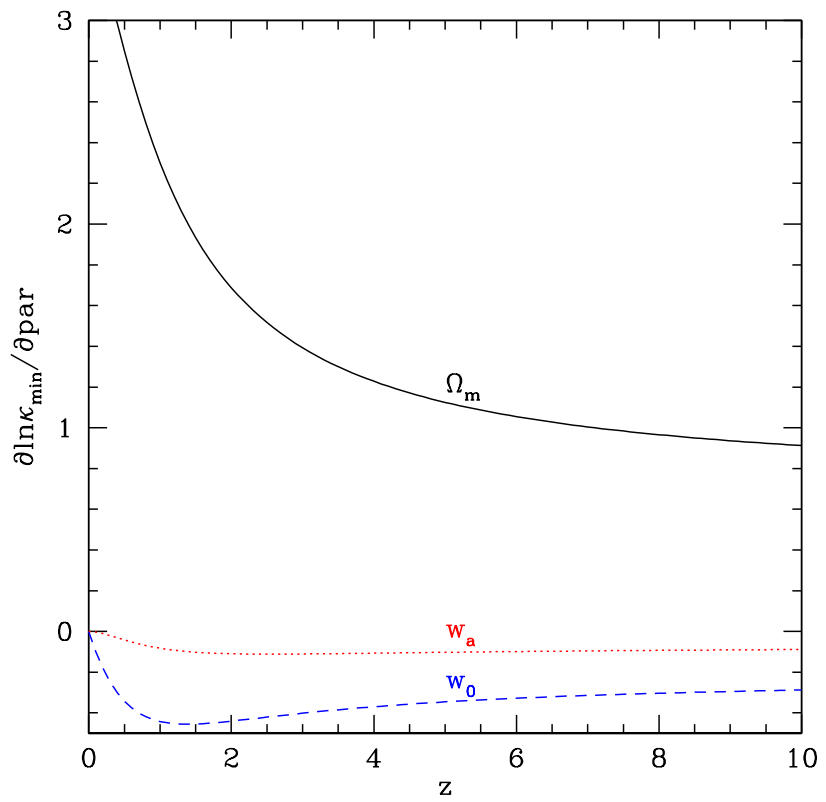

FIG. 3: The Fisher derivative of the logarithm of the observable $\kappa_{\min }$ with respect to the cosmological parameters indicates the sensitivity to the parameters at different redshifts. Similar shapes of curves over a redshift range indicate degeneracy between parameters and relatively little additional information, here occurring for $z>5$.

The main sensitivity is to the matter density, at $z=1.5$ some 4 times more than to $w_{0}$ and 19 times more than to $w_{a}$. At high redshift the sensitivity declines and the curves become similar in shape, indicating substantial degeneracy. Thus we do not anticipate that sources at

\footnotetext{
${ }^{2}$ I am particularly grateful to Daniel Holz for this idea
} 
$z \gtrsim 5$ will contribute effectively to cosmological model estimation. Since such distant sources would be difficult to observe accurately and in sufficiently large numbers (and lines of sight possessing the minimum convergence, i.e. no matter along the entire path, become increasingly rare), it is fortunate that the sensitivity indicates no need for such deep surveys.

Proceeding further, we investigate the cosmological leverage of $\kappa_{\min }$ as a probe in isolation. Considering measurements of $10 \%$ precision, even 100 data points, every 0.1 in redshift over $z=0.1-10$, do not put tight constraints on dark energy properties; the uncertainty on $w_{0}$ is 0.36 and on $w_{a}$ is 1 . However, this is due to a strong degeneracy among the parameters and if we consider combining $\kappa_{\min }$ measurements with the distance measurements of the standard sirens or candles themselves, the picture is quite different.

The minimum convergences have strong complementarity with the distances and improve the distance constraints substantially. Figure 4 shows that as a complement to supernova (SN) distances out to $z=1.7$ of the quality expected from the SNAP supernova survey, the minimum convergence has essentially the impact of the Planck cosmic microwave background measurements. Here we considered $10 \%$ measurements of $\kappa_{\min }$ every 0.5 in redshift over $z=1-5$. Note that this may be a reasonable redshift range as for lower redshifts lensing effects are weaker and harder to separate from the standard amplitude dispersion, while for higher redshifts the sources may be harder to detect in sufficient numbers to measure robustly the minimum amplification cutoff.

In terms of the parameter uncertainties, adding the $\kappa_{\text {min }}$ measurements tightens constraints on $w_{a}$ by a factor 1.5 and decreases the contour area by a factor 2.7 . An advantage of using $\kappa_{\min }$ is that there is no need to extrapolate the behavior of the dark energy to $z>1000$, as there is for using the CMB constraint. If one does add CMB data as well, then the $w_{a}$ uncertainty decreases by a further $7 \%$ and the area shrinks by a further $29 \%$.

Using the distances measured from the gravitational wave $(\mathrm{GW})$ sirens themselves, rather than from supernovae, does not give as strong constraints if we assume that their accuracy is limited to $1 \%$ over the same redshift range as we use $\kappa_{\min }$ - remember that lensing degrades the precision of these high redshift distance determinations. Use of GW distances and $\kappa_{\min }$ gives contours outside the range shown in Fig. 44(though lower redshift GW distances would tighten the constraints). Adding GW distances to the previous set of SN distances and $\kappa_{\text {min }}$ improves $w_{0}$ estimation by $15 \%, w_{a}$ by $7 \%$ and the area shrinks by $20 \%$, as shown in Fig. 4. Thus, SN distances (or low redshift GW distances) are important complements.

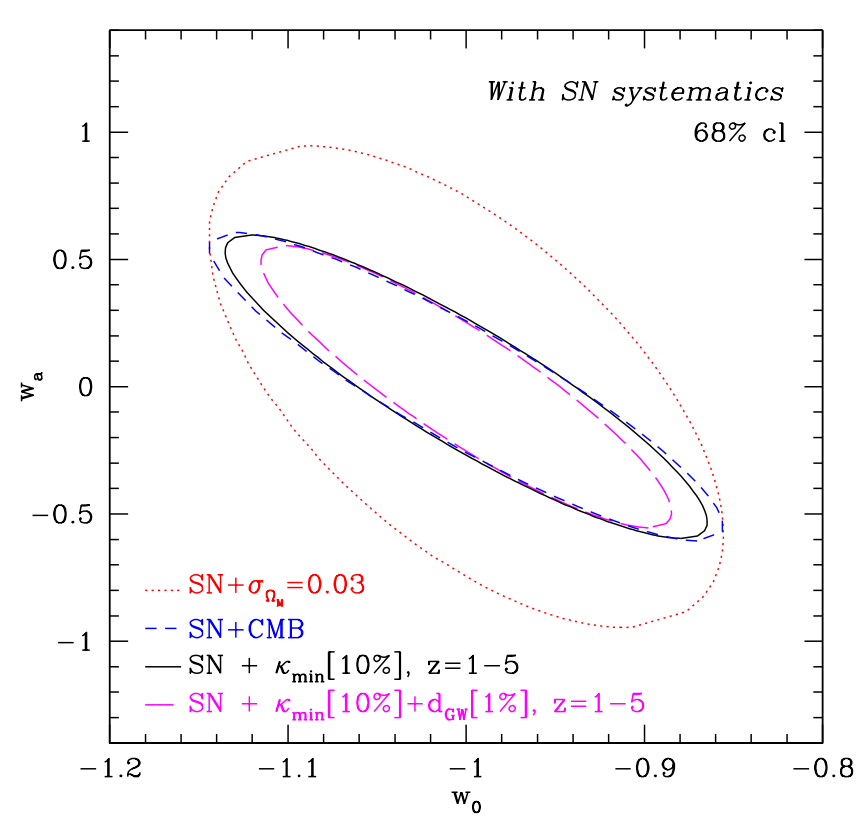

FIG. 4: Minimum amplification has good complementarity with distance probes for determining the dark energy equation of state, shown here as the $1 \sigma$ joint confidence contours in $w_{0}$, $w_{a}$. It is closely equivalent in its constraint leverage to CMB data, but without extrapolating to the early universe.

\section{Lensing Dispersion as Growth Probe}

Note that both the standard siren/candle distances and the minimum amplification of the lensing probability function measured through the siren/candle amplitude distribution are geometric probes of the cosmic expansion history. However, the lensing itself arises from mass inhomogeneities and so contains information on the growth history of structure in the universe. This last use has been discussed by, e.g., [12, 13, 14] as a means of measuring the current strength of mass fluctuations, $\sigma_{8}$. Basically, the width of the lensing induced dispersion is a weighted function of the mass power spectrum.

Here we briefly outline how measurements of the lensing dispersion as a function of redshift probe the linear density growth factor, $g(z)=(1+z)\left[\delta \rho_{m} / \rho_{m}\right](z)$. The mass power spectrum is proportional to the linear growth factor squared and the transfer function. A standard approximation, which will need to be improved for future precision cosmology, is that the transfer function is insensitive to deviations from the LCDM model; thus the lensing dispersion may allow determination of the linear growth (with no galaxy bias entering). If successful, this means that by measuring distances through standard sirens/candles, one actually has three probes of cosmology: 1) the mean amplitude gives a geometric measure of the distance, 2) the minimum amplification gives a complementary geometric measure consisting of distance 
ratios, and 3) the dispersion encodes the growth history through the linear growth factor.

Defining the width or dispersion of the lensing probability distribution function (pdf) is nontrivial. In an effort to develop understanding of lensing pdf's suitable for the high redshifts and precisions needed for next generation surveys, [15] has investigated the hyperLandau (HL) form

$$
P(\mu)=\frac{2^{-s}}{\sigma_{2} \Gamma(s)} e^{-\frac{1}{2}\left[\frac{\mu-\mu_{\star}}{\sigma_{1}}+e^{\left.-\frac{\mu-\mu_{\star}}{\sigma_{2}}\right]}\right.}
$$

with some success. This is a three (or fewer) parameter form, with $\sigma_{1}, \sigma_{2}, \mu_{\star}$, where $\Gamma(s)$ is the gamma function and $s=\sigma_{2} /\left(2 \sigma_{1}\right)$. The variance is proportional to $\sigma_{2}^{2}$ and when $s$ is independent of redshift then the width of the distribution as a function of redshift scales as $\sigma_{2}(z)$. This can be calibrated against ray tracing simulations and may allow the growth factor $g(z)$, to which it should be proportional, to be extracted, though further work is required to verify this ${ }^{3}$.

If this does allow measurement of $g(z)$, then GW standard sirens or SN standard candles provide a triple probe of dark energy, including both the expansion and the growth histories. Figure 5 shows that $10 \%$ measurements of the growth factor $g(z)$ over the same redshift range as $\kappa_{\text {min }}$ does not add much knowledge in $w_{0}, w_{a}$ - but recall that one of the points of combining expansion probes with growth probes is to provide a crosscheck and a window on gravity beyond Einstein, e.g. through the gravitational growth index $\gamma$ [17], not just $w_{0}, w_{a}$.

In order to see significant improvements in the $w_{0}-w_{a}$ plane, one requires $2 \%$ accuracy in the $g(z)$ measurements from the width of the lensed amplitude distribution, which may be challenging. If achievable, this delivers $12 \%$ improvement in determining $w_{0}, 27 \%$ in $w_{a}$, and a factor 1.51 in the contour area. From Fig. 5 we see that we also require $2 \%$ accuracy in measuring $g(z)$ to roughly substitute for supernovae distances, i.e. to have a purely gravitational wave standard siren constraint, though this also requires either precision low redshift distance or Hubble constant measurements.

\section{CONCLUSIONS}

Standardized siren or candle measurements of the mean distance-redshift relation provide precise cosmological parameter estimation, but their full information is even richer. The distribution in the binary black hole

\footnotetext{
${ }^{3}$ Note that one may actually use the linearly scaled variable $u=$ $\left(\mu-\mu_{\min }\right) /\left(1-\mu_{\min }\right)$ to improve the universality, i.e. cosmology independence, of the pdf [16]. Also, the steep dropoff of the form (12) on the deamplification side gives support to $\kappa_{\min }$ being reasonably precisely measurable.
}

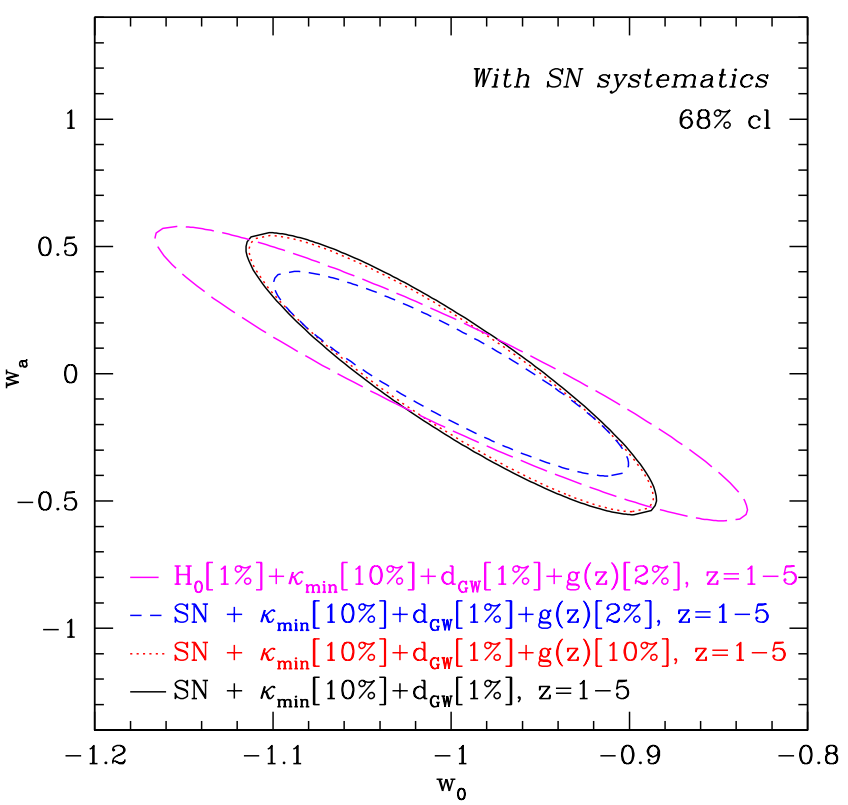

FIG. 5: Standard siren or candle measurements also probe the growth history through the width of the lensed amplitude distribution. Accuracy approaching $2 \%$ on the linear growth factor is required for significant additional constraints on the dark energy equation of state, but less stringent growth estimation may provide important crosschecks or tests of extended gravity. Here the solid curve corresponds to the longdashed curve of Fig. 4, without growth information.

gravitational wave strain amplitude or supernova luminosity carries with it characteristics reflecting the propagation along the line of sight. In particular, this distribution has a physically precise lower boundary due to the minimum convergence, which involves a distance ratio, providing a further geometric measurement of the cosmology.

From the full, long known definition of the minimum convergence or maximum demagnification we show that a common approximation induces a substantial bias as measurements move toward higher redshifts and better precision. We explore several observational issues with using minimum amplification as a practical cosmological probe, suggesting that gravitational wave standard sirens might enable this method if sufficient numbers of sirens can be detected with precise redshifts.

Along with a new probability distribution function for lensing amplification, we discuss how the width of the distribution might be used as a measure of the mass power spectrum or linear growth factor. From a Fisher analysis it appears that the method would need to be developed to allow $2 \%$ accuracy on the lensing dispersion for a significant gain in cosmological constraints, but weaker precision could still provide useful crosschecks or consistency tests of gravity.

Substantial work needs to be done to realize the 
promises of the lensing distribution information for precision cosmology. In particular, large ray tracing simulations are needed to verify and accurately calibrate the minimum detectable convergence and distribution width.

While challenging, nevertheless it is exciting that distance measurements of gravitational wave standard sirens and possibly supernova standardized candles can act as a triple probe of cosmology and dark energy: 1) the mean amplitude (unaffected by lensing) gives a geometric measure of the distance, 2) the minimum amplification gives a complementary geometric measure consisting of distance ratios, and 3) the dispersion encodes the growth history through the linear growth factor. If this potential can be realized, distance-redshift measurements of sirens or candles can provide probes of both the cosmic expansion and growth histories.

\section{Acknowledgments}

I thank Daniel Holz for useful discussions and the T6 group at Los Alamos National Laboratory for hospitality. This work has been supported in part by the Director, Office of Science, Department of Energy under grant DEAC02-05CH11231.

\section{APPENDIX A: AVERAGING INHOMOGENEITY}

One might wonder whether the commonly used approximation Eq. (8) is sufficiently accurate for use along arbitrary lines of sight, in comparison to the expression for arbitrary convergence $\kappa$, Eq. (3). This is an important point since $\kappa$ plays a central role in all gravitational lensing calculations. The answer, fortunately, is that ex-

${ }^{4}$ In the weak lensing case specifically this holds nearly trivially as then $\kappa \ll 1$ and so $\alpha \approx 1$ (cf. Eq. 4), so $r_{\alpha} \approx r_{\mathrm{FRW}}$. cept for the special situation when one considers the particular lines of sight emptied of matter - i.e. the minimum convergence treated in the main text - use of the FRW distances is an excellent approximation and standard lensing formulas hold ${ }^{4}$.

Formally, one might write Eq. (4) as

$$
\kappa=\frac{3}{2} \Omega_{m} \int_{1}^{1+z} d y \frac{y^{2}}{H(y) / H_{0}} \delta(y) r_{\alpha}(y) \frac{r_{\mathrm{FRW}}(y, 1+z)}{r_{\mathrm{FRW}}(1+z)}
$$

nearly the usual gravitational lensing form, except that instead of $r_{\mathrm{FRW}}(y)$ we have $r_{\alpha}$ evaluated for $\alpha=\langle 1+$ $\left.\delta\left(y^{\prime}<y\right)\right\rangle$, where $\delta=\delta \rho_{m} / \rho_{m}$. That is, $r(y)$ depends on the energy-momentum density within the beam at every redshift between source and observer, giving rise to a weighted smoothness parameter.

This is basically a question of line of sight depth averaging over inhomogeneities to recover a FRW universe and is treated in detail in [18] (along with other types of averaging and their possibly different results). Summarizing, light propagating along a random line of sight will experience both underdense and overdense regions. For a typical inhomogeneity coherence scale $l$, there will be $L / l$ regions over a path length $L$ and the deviations from FRW behavior diminish with depth. This is made more precise in [18] where it is shown that the $Q$ terms in Eq. (2) correspond to $\nabla^{2} \phi$, where $\phi$ is the gravitational potential. Then for $\phi \sim \mathcal{O}\left(\epsilon^{2}\right)$, the correction to distances, $r_{\langle\alpha\rangle}-r_{\mathrm{FRW}}$, is $\sim \epsilon^{2} /(l / L)$, which must be much less than one for a globally FRW universe (see [19]).

The exception to this is when considering special lines of sight such that $\delta$ is constrained, e.g. $\delta=-1$ for the minimum convergence. However, lensing generically involves the natural fluctuations of density along the line of sight and thus can robustly use FRW distances.
[1] C.C. Dyer \& R.C. Roeder, Ap. J. Lett 174, L115 (1972)

[2] D.E. Holz \& S.A. Hughes, Ap. J. 629, 15 (2005) arXiv:astro-ph/0504616

[3] N. Dalal, D.E. Holz, S.A. Hughes, B. Jain, Phys. Rev. D 74, 063006 (2006) arXiv:astro-ph/0601275

[4] R.K. Sachs, Proc. Roy. Soc. London A 264, 309 (1961)

[5] C.C. Dyer \& R.C. Roeder, Ap. J. Lett 180, L31 (1973)

[6] E.V. Linder, A\&A 206, 190 (1988)

[7] P. Schneider \& A. Weiss, Ap. J. 327, 526 (1988)

[8] P. Schneider, J. Ehlers, E.E. Falco, Gravitational Lenses (Springer, 1992)

[9] S. Weinberg, Ap. J. Lett. 208, L1 (1976)

[10] D.E. Holz \& E.V. Linder, Ap. J. 631, 678 (2005) arXiv:astro-ph/0412173

[11] G. Aldering et al., Astropart. Phys. 27, 313 (2007) arXiv:astro-ph/0607030
[12] J.A. Frieman, Commun. Astrop. 18, 323 (1996) arXiv:astro-ph/9608068

[13] U. Seljak \& D.E. Holz, A\&A 351, L10 (1999) arXiv:astro-ph/9910482

[14] S. Dodelson \& A. Vallinotto, Phys. Rev. D 74, 063515 (2006) arXiv:astro-ph/0511086

[15] D.E. Holz \& E.V. Linder, in preparation

[16] D. Munshi \& B. Jain, MNRAS 318, 109 (2000) arXiv:astro-ph/9911502

[17] D. Huterer \& E.V. Linder, Phys. Rev. D 75, 023519 (2007) arXiv:astro-ph/0608681

[18] E.V. Linder, arXiv:astro-ph/9801122

[19] M.W. Jacobs, E.V. Linder, R.V. Wagoner, Phys. Rev. D 45, 3293 (1992); M.W. Jacobs, E.V. Linder, R.V. Wagoner, Phys. Rev. D 48, 4623 (1993) 